\title{
Multiple emission line components in detached post-common-envelope binaries ${ }^{\star}$ (Research Note)
}

\author{
C. Tappert ${ }^{1}$, B. T. Gänsicke ${ }^{2}$, A. Rebassa-Mansergas ${ }^{1}$, L. Schmidtobreick ${ }^{3}$, and M. R. Schreiber ${ }^{1}$
}

\author{
1 Departamento de Física y Astronomía, Facultad de Ciencias, Universidad de Valparaíso, Av. Gran Bretaña 1111, Valparaíso, Chile \\ e-mail: [ctappert,arebassa]@dfa.uv.cl; matthias.schreiber@uv.cl \\ 2 Department of Physics, University of Warwick, Coventry CV4 7AL, UK \\ e-mail: Boris.Gaensicke@warwick.ac.uk \\ 3 European Southern Observatory, Casilla 19001, Santiago 19, Chile \\ e-mail: 1schmidt@eso.org
}

Received 4 March 2011 / Accepted 5 May 2011

\begin{abstract}
Motivated by the recent discovery of an $\mathrm{H} \alpha$ emission line component originating close to the white dwarf primary in the post-commonenvelope binary (PCEB) LTT 560, we have undertaken a spectroscopic snapshot survey on 11 short-period $\left(P_{\text {orb }}<6\right.$ h) PCEBs using FORS2. We have found multi-component $\mathrm{H} \alpha$ emission line profiles in six of our targets, indicating that multiple $\mathrm{H} \alpha$ emission sites are rather common in short-period PCEBs. The underlying physical mechanisms, however, can be fundamentally different. In our sample we find one system where the extracted radial velocities of two identified components indicate that both originate in the late-type secondary star, where one is caused by irradiation from the hot white dwarf primary and the other by stellar activity. Another object presents two components that are located on opposite sides of the centre-of-mass, suggesting a case similar to LTT 560, i.e. that accretion of stellar wind from the secondary star produces $\mathrm{H} \alpha$ emission on, or close to, the white dwarf.
\end{abstract}

Key words. binaries: close - line: profiles - white dwarfs

\section{Introduction}

The recipe for forming a close interacting binary star such as a cataclysmic variable contains the common envelope (CE) phase as a crucial ingredient. Here the more massive star of the detached progenitor binary system expands in the course of its nuclear evolution, fills its Roche lobe, and transfers mass at high rates to its low-mass companion, usually a late-type (K-M) main-sequence star. The resulting CE provides enhanced friction, thus causing an accelerated reduction of the binary separation. For details on the CE phase see Webbink (2008) and Taam \& Ricker (2010), and references therein.

The resulting post-common-envelope binary (PCEB) is a detached white dwarf/late-type dwarf system with an orbital period of a few days or less. Its binary separation continues to decrease due to angular momentum loss driven by magnetic braking and gravitational wave radiation until eventually the Roche lobe of the secondary star comes into contact with the stellar surface, thus starting mass transfer via Roche lobe overflow.

Since the PCEB is still a detached system one would expect the $\mathrm{H} \alpha$ emission line - if present at all - to have a simple, single-component profile originating in the secondary star and produced by stellar activity or by irradiation of a sufficiently hot white dwarf primary. However, there are a number of systems known that present a more complex line profile. For example, in some PCEBs with a hot white dwarf primary, non-LTE effects produce an inverted core in the irradiation induced emission

\footnotetext{
$\star$ Based on observations made at ESO telescopes (proposals 383.D0430, 384.D-0596).
}

lines that give them a double-peaked profile (Barman et al. 2004). As an example see the recent study of NN Ser by Parsons et al. (2010). The PCEB QS Vir shows a complex line profile composed of at least three components that led O'Donoghue et al. (2003) to suspect an accretion disc as one of the origins, while Parsons et al. (2011) suggest stellar prominences as the mechanism behind it. Finally, the $\mathrm{H} \alpha$ line in the system LTT 560 exhibits two exactly anti-phased components. One component originates in the secondary star and is related to stellar activity, while the other comes from a chromosphere/corona on the white dwarf as the probable result of accretion of stellar wind from the companion star (Tappert et al. 2007, 2011).

Systems of this last type present an attractive possibility to determine a full set of parameters (i.e., masses, inclination, binary separation), since measuring the gravitational redshift from the $\mathrm{H} \alpha$ component on the white dwarf yields its mass, and thus breaks the parameter degeneracy of the orbital equations. Ultraviolet spectra presented by Kawka et al. (2008) proved that ongoing wind accretion in detached PCEBs is a common phenomenon. However, LTT 560 so far represents the only known example where the signature of such accretion is observed as an $\mathrm{H} \alpha$ emission line component located on the white dwarf. Consequently, it is unclear under what conditions such chromospheric emission on the white dwarf will develop. One may assume that it is related to the mass-transfer/accretion rate. PCEBs that are already comparatively close to contact and/or contain an active secondary star with a strong stellar wind should thus represent good candidates. The probability of fulfilling both, i.e. having late-type secondary star with a large Roche-lobe 
Table 1. Log of observations.

\begin{tabular}{|c|c|c|c|c|c|c|c|c|c|c|}
\hline (1) & (2) & (3) & (4) & (5) & (6) & (7) & (8) & (9) & (10) & (11) \\
\hline SDSS & date & $\begin{array}{l}\text { HJD } \\
\text { [d] }\end{array}$ & $n$ & $\begin{array}{l}t_{\exp } \\
{[\mathrm{s}]}\end{array}$ & $\begin{array}{l}\Delta t \\
{[\mathrm{~h}]}\end{array}$ & $\begin{array}{l}\Delta \varphi \\
\text { orbits }\end{array}$ & $\begin{array}{l}P_{\text {orb }} \\
{[\mathrm{h}]}\end{array}$ & $\begin{array}{l}T_{\mathrm{WD}} \\
{\left[10^{3} \mathrm{~K}\right]}\end{array}$ & MS & $\begin{array}{l}r \\
{[\mathrm{mag}]}\end{array}$ \\
\hline J005245.11-005337.2 & $2009-10-17$ & 2455122 & 3 & 900 & 0.52 & 0.19 & 2.73 & 16 & M4 & 19.1 \\
\hline J015225.38-005808.5 & $2009-10-17$ & 2455122 & 10 & 240 & 0.69 & 0.32 & 2.17 & 9 & M6 & 17.7 \\
\hline J023804.39-000545.7 & $2009-10-17$ & 2455122 & 4 & 660 & 0.58 & 0.11 & 5.08 & 22 & M3 & 18.7 \\
\hline J030308.35+005444.1 & $2009-10-17$ & 2455122 & 7 & 360 & 0.66 & 0.21 & 3.20 & - & M4 & 18.1 \\
\hline $\mathrm{J} 141134.70+102839.7$ & 2009-04-15 & 2454937 & 3 & 900 & 0.52 & 0.13 & 4.02 & 30 & M3 & 19.1 \\
\hline $\mathrm{J} 152933.25+002031.2$ & 2009-04-15 & 2454937 & 4 & 660 & 0.58 & 0.15 & 3.95 & 14 & M5 & 18.3 \\
\hline $\mathrm{J} 155904.62+035623.4$ & 2009-04-15 & 2454937 & 4 & 660 & 0.58 & 0.26 & 2.27 & 49 & - & 18.6 \\
\hline J211205.31+101427.9 & $2009-06-20$ & 2455003 & 4 & 660 & 0.58 & 0.27 & 2.17 & 20 & M6 & 18.4 \\
\hline $\mathrm{J} 212320.74+002455.5$ & $2009-05-25$ & 2454977 & 6 & 900 & 1.39 & 0.39 & 3.58 & 13 & M6 & 19.4 \\
\hline J213218.11+003158.8 & $2009-05-26$ & 2454978 & 7 & 360 & 0.66 & 0.12 & 5.33 & 16 & M4 & 18.2 \\
\hline J221616.59+010205.6 & $2009-05-26$ & 2454978 & 7 & 360 & 0.66 & 0.13 & 5.05 & 12 & M5 & 18.0 \\
\hline
\end{tabular}

Notes. Columns: (1) target's SDSS designation; (2) start of night of observation; (3) Heliocentric Julian Day; (4) number of exposures; (5) exposure time; (6) covered time range; (7) covered fraction of orbital cycle; (8) orbital period; (9) approximate temperature of the white dwarf; (10) spectral type of the secondary star; (11) magnitude in Sloan $r$.

Orbital periods were taken from Rebassa-Mansergas et al. (2008) and Nebot Gomez-Moran et al. (in prep.), Cols. (9)-(11) are from Rebassa-Mansergas et al. (2010).

filling factor, is highest in short-period PCEBs, such as LTT 560 $\left(P_{\text {orb }}=3.54 \mathrm{~h}\right)$. We here present a spectroscopic time-series snapshot survey of 11 PCEBs from the Sloan Digital Sky Survey (SDSS; Abazajian et al. 2009, and references therein) with $P_{\text {orb }} \leq 6$ h.

\section{Observations and data reduction}

Spectroscopic time series data were taken with FORS2 (Appenzeller et al. 1998) mounted on UT1 (ANTU) at the ESO-Paranal Observatory. The instrument was used in long-slit mode (LSS) with grism $1200 \mathrm{R}+93$ and filter GG435. The covered spectral range was 5750-7230 , and an $0.4^{\prime \prime}$ slit gave a spectral resolution of $2.3 \AA$. The observations were conducted in service mode with one observing block (OB) of less than $1 \mathrm{~h}$ per target (2 subsequent OBs for the faintest one, J2123). Each OB consisted of a series of 3-10 spectra, depending on the target's brightness and its orbital period. Table 1 presents a summary of the observations.

Data reduction was performed with IRAF, and included the correction with bias and flatfield frames. The spectra were extracted, and wavelength calibration was obtained with arc lamp spectra that were taken once per night. The dispersion solution proved stable over the complete range spanned by the observations, i.e. over several months. The data were not flux calibrated.

Radial velocities of the $\mathrm{H} \alpha$ emission line were measured using single Gaussian functions. In two cases, J1559 and J2216, an additional emission component was sufficiently visible in at least one spectrum to fit a combination of two Gaussians. The resulting full width at half maximum $(F W H M)$ and amplitude of the weaker component were then held fixed to measure the radial velocities in the remaining spectra.

\section{Results}

For six of the eleven systems we find a significantly variable emission line profile that suggests the presence of at least two emission components (Fig. 1). Of the remaining five objects, four are very likely to have a single-component $\mathrm{H} \alpha$ emission, while one system, J1411, needs more data. In the following we review the results for the individual systems.
J1559, J2216: in one or more spectra the two components were resolved enough to fit individual Gaussian functions to each of them. For J1559 the two components move together in phase, but with different amplitudes. The most likely explanation for this behaviour is that both components originate in the mainsequence star, one due to stellar activity, the other irradiation. The latter will be more confined to the white-dwarf facing side, thus be situated closer to the centre-of-mass, and consequently have a lower radial-velocity amplitude. In J1559, this would also be the stronger of the two components. We also note that J1559 contains by far the hottest white dwarf of our sample (Table 1). With $T_{\mathrm{WD}}=49000 \mathrm{~K}$ it has a high probability of an irradiation effect (see Sect. 4).

J2216, on the other hand, shows two equally strong components that move in roughly anti-phased manner. Assuming that one of them is located on the secondary star, the other then would have to develop on the white-dwarf side of the centre-of-mass, suggesting a similar situation as in LTT 560.

J0152, J0303, J2112: while the individual components are not resolved, in all three objects we find a strongly asymmetric line profile in the spectra that are closest to one of the extrema of the radial velocity curve. This is clear evidence that the line profile is composed of multiple components.

J0052: the asymmetry in the first spectrum of a series of three is less notable than in above three systems, but it is doubtlessly there. The radial velocity curve suggests that the three spectra were taken close to conjunction, when emission components that are in phase or anti-phased would have very similar velocities.

J1411: we do not find any significant line profile variation in this object. However, as for J0052, our series consists of only three spectra that were taken close to conjunction as indicated by the radial velocity curve. Additionally, only 0.13 orbital cycles are covered by the spectra.

J0238, J1529, J2123, J2132: all four systems have weak and narrow single-component line profiles. These profiles do present 


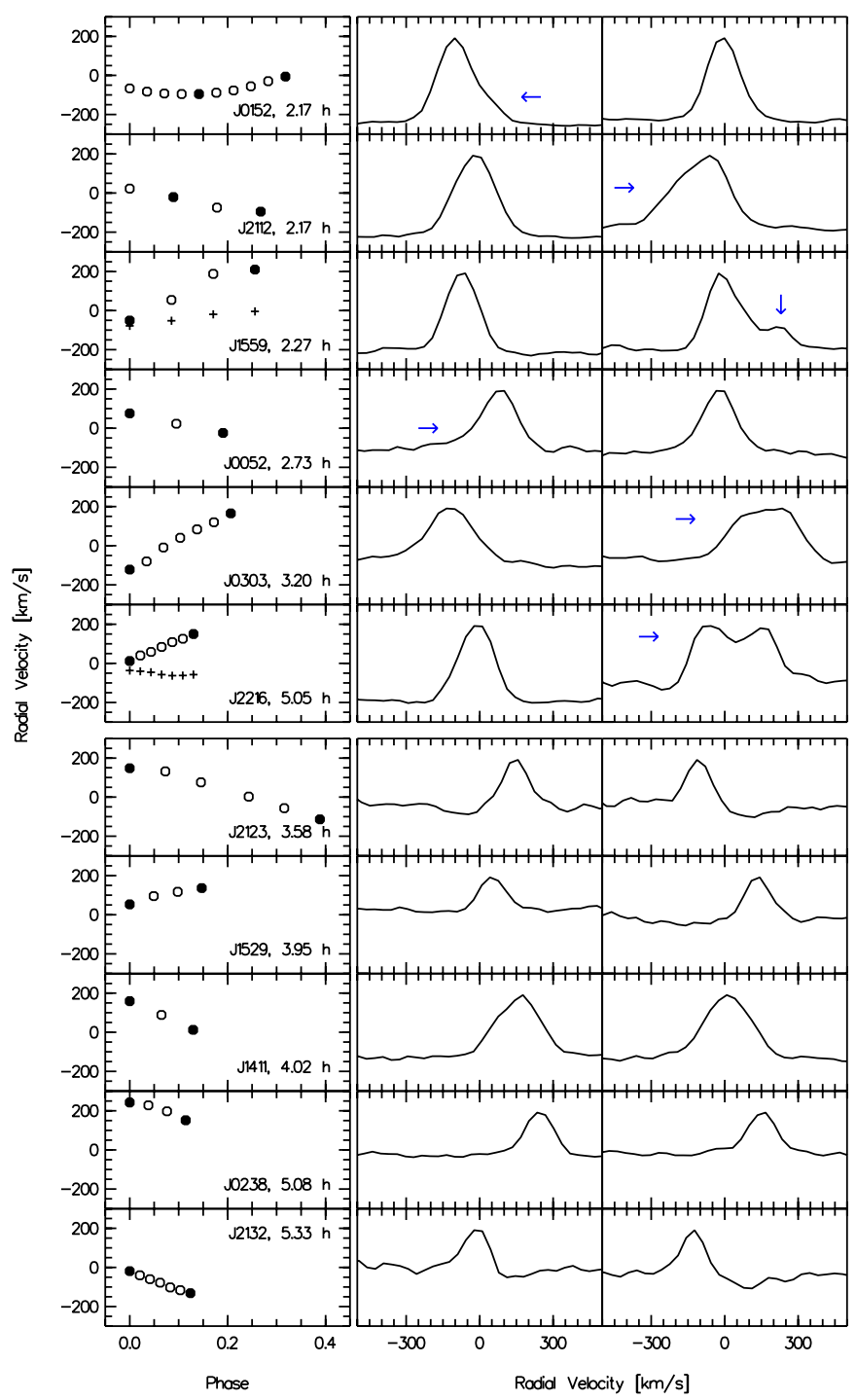

Fig. 1. H $\alpha$ line profiles of the targets, normalised to the intensity maximum. For each object, two examples profiles are shown that correspond to the radial velocities marked as filled circles in the left subpanel. Since the ephemerides are not known with sufficient precision for an extrapolation, the zero point of the orbital phase was arbitrarily set to the first data point of the respective sets. The upper main panel presents the six systems with multiple emission sources, with arrows marking the signature of the weaker component. The lower panel shows the remaining five systems. The radial velocities were measured by fitting a single Gaussian to the line profile, with the exception of J1559 and J2216, where a combination of two Gaussians was used.

some variability, but here it is caused by the superposition of the $\mathrm{H} \alpha$ emission from the secondary with the broader $\mathrm{H} \alpha$ absorption of the white dwarf primary that move in anti-phase.

In Fig. 2 we show the average $F W H M$ of a Gaussian function fit to the series of line profiles of a specific object versus its amplitude. The assumption is that lines composed of more than one component have, on average, a broader profile and, as the multiple components merge and separate with orbital phase, show a larger variation in both intensity and width when fitted with a single Gaussian than genuine single-component lines. While aware of looking at small number statistics, we do note that the two types of systems are clearly separated, with all multiple-component objects having $F W H M_{\text {av }}>100 \mathrm{~km} \mathrm{~s}^{-1}$, while $F W H M_{\text {av }}<90 \mathrm{~km} \mathrm{~s}^{-1}$ for all single-component systems,

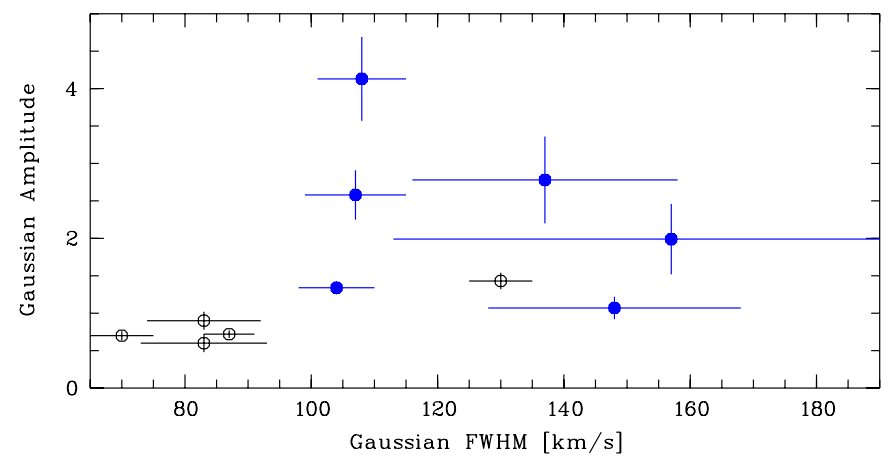

Fig. 2. Average values for $F W H M$ and amplitude of Gaussian fits to the line profile for each system. Here, all spectra were fit with a single Gaussian, regardless of potentially resolved components. The error bars indicate the variability of these parameters over the set of line profiles for a specific system. Filled circles mark objects with multiple components.

with $\mathrm{J} 1411\left(F W H M_{\mathrm{av}}=130 \mathrm{~km} \mathrm{~s}^{-1}\right)$ being the sole exception. Considering the width of the line profile and the small orbital phase coverage that additionally is likely to be close to conjunction, we thus conclude that with the present data we cannot exclude with certainty the presence of multiple emission components in J1411. In this context we note that our spectral resolution of $\sim 2.3 \AA$ translates to $\sim 100 \mathrm{~km} \mathrm{~s}^{-1}$ at $\mathrm{H} \alpha$. This explains the clustering of the single-component systems with $F W H M_{\text {av }}<90 \mathrm{~km} \mathrm{~s}^{-1}$, which thus is simply due to our limited spectral resolution. Consequently the respective line profiles are not resolved, and the real FWHM is probably even lower for those systems.

\section{Discussion}

In a snapshot survey of 11 short-period PCEBs we have found strong evidence of multiple $\mathrm{H} \alpha$ emission sources in six systems. Due to the limited phase coverage of our spectroscopy it is not possible to determine the location within the binary system of any of those sources. One might expect that at least one of them is located on the secondary star, with the underlying mechanism either stellar activity or irradiation by the white dwarf primary. However, in J1559 we find two components that move in phase but with different amplitudes, which can be interpreted as both these effects being present at the same time. In contrast, $\mathbf{J} 2216$, the only other system where we can actually measure the velocities of the individual components, presents two approximately anti-phased emission components, which then should come from opposite sides of the centre-of-mass.

These two examples already highlight that very different physical mechanism are underlying the multiple $\mathrm{H} \alpha$ components in PCEBs. So far, we can discern two fundamentally different scenarios for emission line profiles that deviate from those in single late-type dwarfs. On the one hand, irradiation from a hot white dwarf leads to non-LTE effects (such as in NN Ser; Parsons et al. 2010) or to a two-component profile that combines the recombination emission with the one from stellar activity, such as in J1559. On the other hand, mass transfer can lead to prominences (QS Vir; Parsons et al. 2011) or a white-dwarf corona (LTT 560; Tappert et al. 2011).

Despite the complex phenomenology one may ask if it is possible to find a common property that would at least allow to discern those PCEBs with multiple emission sources from those with single components. To explore the parameter space we 
Table 2. Comparison of WD/MS PCEBs with $P_{\text {orb }}<1 \mathrm{~d}$ that have time-series spectroscopic data published at sufficient high spectral resolution to examine the $\mathrm{H} \alpha$ emission line profile for the presence of multiple components.

\begin{tabular}{|c|c|c|c|c|c|c|}
\hline (1) & (2) & (3) & (4) & $(5)$ & $(6)$ & (7) \\
\hline object & $\begin{array}{l}P_{\text {orb }} \\
{[\mathrm{h}]}\end{array}$ & $\begin{array}{l}T_{\mathrm{WD}} \\
{\left[10^{3} \mathrm{~K}\right]}\end{array}$ & MS & $n_{\mathrm{H} \alpha}$ & $\mathrm{H} \alpha_{\mathrm{MS}}$ & references \\
\hline J0152 & 2.17 & 9 & M6 & 2 & - & this paper \\
\hline $\mathrm{J} 2112$ & 2.17 & 20 & M6 & 2 & - & this paper \\
\hline J1559 & 2.27 & 49 & - & 2 & $\mathrm{a}+\mathrm{i}$ & this paper \\
\hline HR Cam & 2.47 & 19 & - & (1) & $\mathrm{i}$ & Bergeron et al. (1992), Marsh \& Duck (1996), Maxted et al. (1998) \\
\hline J0052 & 2.73 & 16 & M4 & 2 & _ & this paper \\
\hline HS $2237+8154$ & 2.98 & 12 & M3.5 & 2 & a & Gänsicke et al. (2004) \\
\hline NN Ser & 3.12 & 57 & M4 & (1) & $\mathrm{i}$ & Haefner (1989), Haefner et al. (2004), Parsons et al. (2010) \\
\hline J0303 & 3.20 & - & M4 & 2 & - & this paper \\
\hline LTT 560 & 3.54 & 7 & M5.5 & 2 & a & Tappert et al. (2007), Tappert et al. (2011) \\
\hline $\mathrm{J} 2123$ & 3.58 & 13 & M6 & 1 & - & this paper \\
\hline QS Vir & 3.62 & 14 & M4 & 3 & a & Kawka et al. (2002), O’Donoghue et al. (2003), Parsons et al. (2011) \\
\hline J1529 & 3.95 & 14 & M5 & 1 & - & this paper \\
\hline J1411 & 4.02 & 30 & M3 & $1 ?$ & - & this paper \\
\hline BPM 71214 & 4.85 & 17 & M2.5 & 1 & a & Kawka et al. (2002), Kawka \& Vennes (2003) \\
\hline J2216 & 5.05 & 12 & M5 & 2 & - & this paper \\
\hline J0238 & 5.08 & 22 & M3 & 1 & - & this paper \\
\hline J2132 & 5.33 & 16 & M4 & 1 & - & this paper \\
\hline HS $1857+5144$ & 6.38 & 85 & M5 & (1) & $\mathrm{i}$ & Aungwerojwit et al. (2007) \\
\hline CC Cet & 6.89 & 26 & M5 & 1 & $\mathrm{i}$ & Saffer et al. (1993), Somers et al. (1996) \\
\hline RR Cae & 7.30 & 8 & M4 & 1 & a & Bruch \& Diaz (1998), Bruch (1999), Maxted et al. (2007) \\
\hline WD 1042-690 & 8.06 & 21 & - & 1 & a & Bragaglia et al. (1995), Morales-Rueda et al. (2005) \\
\hline DE CVn & 8.74 & 8 & M3 & 1 & a & van den Besselaar et al. (2007) \\
\hline V471 Tau & 12.50 & 35 & $\mathrm{~K} 2$ & 1 & - & $\begin{array}{l}\text { Nelson \& Young (1970), Young \& Nelson (1972); } \\
\text { Gilmozzi \& Murdin (1983); Bois et al. (1991) }\end{array}$ \\
\hline HZ 9 & 13.54 & 17 & M4.5 & 1 & a & Lanning \& Pesch (1981), Bleach et al. (2002), Schreiber \& Gänsicke (2003) \\
\hline UZ Sex & 14.33 & 20 & M4 & 1 & a & Saffer et al. (1993), Kepler \& Nelan (1993), Bruch \& Diaz (1999) \\
\hline EG UMa & 16.03 & 13 & M4 & 1 & a & Bleach et al. (2000), Bleach et al. (2002) \\
\hline WD 2009+622 & 17.78 & 26 & M5.5 & 1 & $\mathrm{i}$ & Bergeron et al. (1992), Morales-Rueda et al. (2005), Farihi et al. (2005) \\
\hline HS $1136+6646$ & 20.06 & 70 & K5.5 & (1) & $\mathrm{i}$ & Sing et al. (2004) \\
\hline
\end{tabular}

Notes. Columns: (2) orbital period; (3) approximate temperature of the white dwarf; (4) spectral type of the main-sequence star; (5) number of $\mathrm{H} \alpha$ emission components, a "1" in brackets marks non-LTE effects; (6) origin of the H $\alpha$ component from the main-sequence star, either stellar (a)ctivity or (i)rradiation from the white dwarf.

enlarged the sample by taking the PCEB lists of Morales-Rueda et al. (2005) and Shimansky et al. (2006), examined the published data on these systems, and included HS $1857+5144$ (Aungwerojwit et al. 2007). Since our main interest concerns potential precataclysmic variables such as LTT 560, we limited the sample to PCEBs with $P_{\text {orb }}<1 \mathrm{~d}$ that contain a white dwarf as primary component.

The systems with data that allow an examination of the $\mathrm{H} \alpha$ emission line profile are presented in Table 2. Apart from the six PCEBs with multiple emission components from the present survey and the known objects LTT 560 and QS Vir, we find one other such system (HS 2237+8154; Gänsicke et al. 2004). While it is not explicitly mentioned by the authors, the trailed spectrum of the $\mathrm{H} \alpha$ emission line (their Fig. 8) clearly shows the presence of both a high-amplitude and a low-amplitude component that are roughly anti-phased. For the system HS 1136+6646 Sing et al. (2004) mention an accretion disc as the possible mechanism behind the double-peaked $\mathrm{H} \alpha$ profile, but the extremely hot white dwarf primary instead suggests a non-LTE effect.

Table 2 includes parameters that might have an influence on the number of emission sources. It is clear that these have to be such that they facilitate the interaction between the two stellar components. A short orbital period basically equals a small binary separation (with the limited mass ranges involved here), and a high white-dwarf temperature increases the probability of an irradiation effect or even a non-LTE phenomenon ${ }^{1}$. Additionally the spectral type of the secondary star is broadly correlated with the strength of a stellar-activity induced $\mathrm{H} \alpha$ emission line (e.g., Berger 2006), although with the very limited range of spectral types in our sample, this should not be a major factor. Other potentially important parameters are the magnetic field strength of the white dwarf and the Roche-lobe filling factor of the secondary star. While there is unfortunately no such data available for most systems, we note that there is at least no positive detection of a strongly magnetic white dwarf in any of the objects in Table 2. Also, while for PCEBs that eventually evolve into cataclysmic variables one expects to find nearly Roche-lobe filling secondaries anywhere in an orbital period range roughly from 1.5 to $10 \mathrm{~h}$, the probability of a large Roche-lobe filling factor will be higher for shorter orbital periods.

We can see in Fig. 3 that these basic assumptions are confirmed. PCEBs with multiple emission components tend to cluster at short orbital periods, and those with single components are mostly confined to long orbital periods, $>4.5 \mathrm{~h}$. In fact, with the exception of non-LTE objects we do not find single-component PCEBs below $P_{\text {orb }}=3.5 \mathrm{~h}$. Furthermore, irradiation effects dominate at $T_{\mathrm{WD}}>25000 \mathrm{~K}$ and do not appear to play any role for

\footnotetext{
1 A notable exception is the system HR Cam, which shows nonLTE features (Maxted et al. 1998), but does not contain a particularly hot white dwarf ( $T_{\mathrm{WD}} \sim 19000 \mathrm{~K}$; Bergeron et al. 1992)
} 


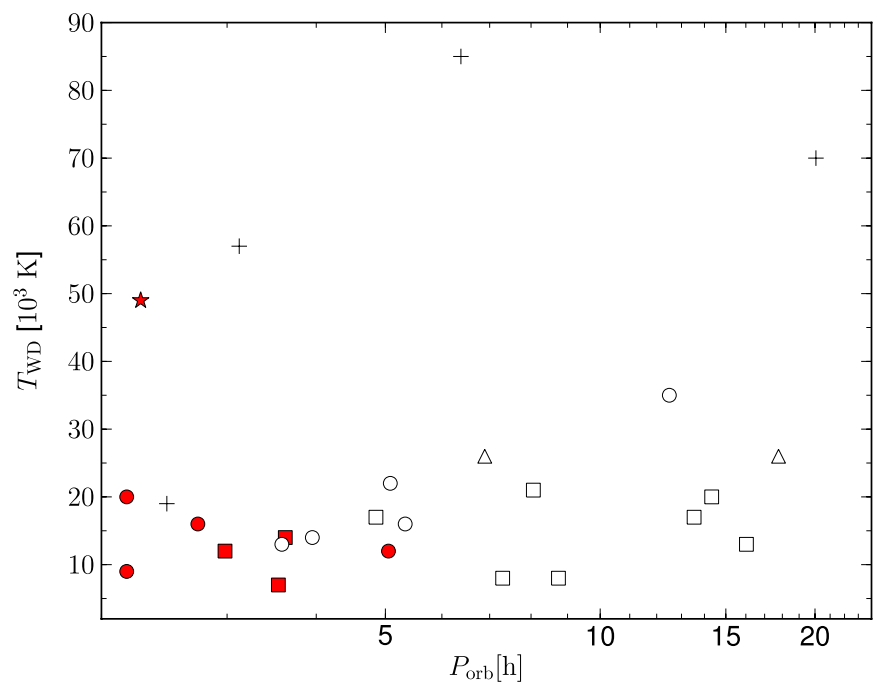

Fig. 3. Orbital period (in logarithmic scale) vs. white-dwarf temperature for the systems in Table 2. Filled symbols mark PCEBs with multiple $\mathrm{H} \alpha$ emission components, open symbols those with single components. The shape of the symbol indicates the type of emission from the secondary star: irradiation (triangle), activity (square), or yet unknown (circle). J1559 is marked by a star, since it probably presents both effects. Objects that show non-LTE effects are indicated by crosses. The plot does not include the systems J0303 ( $T_{\mathrm{WD}}$ unknown) and J1411 (uncertain number of emission components).

$T_{\mathrm{WD}}<20000 \mathrm{~K}$. However, these limits have to be taken with caution since with the available data the danger of overinterpreting small number statistics is all too present.

For a significant fraction of the systems in our sample the origin of the emission from the secondary star is not known, and for the vast majority of the PCEBs with multiple emission sources we still do not have any information on the mechanism behind that phenomenon. In fact, so far the origin of both components has been firmly established only for LTT 560 (Tappert et al. 2011). The present work should therefore encourage more detailed studies, since it is clear that the presence of multiple emission components in PCEBs is not an isolated, but rather a common phenomenon. On the basis of the currently present data we can conclude that the best place to look for it are short-period PCEBs $\left(P_{\text {orb }}<4 \mathrm{~h}\right)$ with a white dwarf primary that is not too $\operatorname{hot}\left(T_{\mathrm{WD}}<20000 \mathrm{~K}\right)$.

Acknowledgements. We thank the referee, John Thorstensen, for helpful comments. A.R.M. acknowledges financial support from Fondecyt in the form of grant number 3110049. This study has made extensive use of NASA's Astrophysics Data System Bibliographic Services. IRAF is distributed by the National Optical Astronomy Observatories.

\section{References}

Abazajian, K. N., Adelman-McCarthy, J. K., Agüeros, M. A., et al. 2009, ApJS, 182,543

Appenzeller, I., Fricke, K., Fürtig, W., et al. 1998, The Messenger, 94, 1

Aungwerojwit, A., Gänsicke, B. T., Rodríguez-Gil, P., et al. 2007, A\&A, 469, 297

Barman, T. S., Hauschildt, P. H., \& Allard, F. 2004, ApJ, 614, 338

Berger, E. 2006, ApJ, 648, 629

Bergeron, P., Saffer, R. A., \& Liebert, J. 1992, ApJ, 394, 228

Bleach, J. N., Wood, J. H., Catalán, M. S., et al. 2000, MNRAS, 312, 70

Bleach, J. N., Wood, J. H., Smalley, B., \& Catalán, M. S. 2002, MNRAS, 335, 593

Bois, B., Mochnacki, S. W., \& Lanning, H. H. 1991, AJ, 102, 2079

Bragaglia, A., Renzini, A., \& Bergeron, P. 1995, ApJ, 443, 735

Bruch, A. 1999, AJ, 117, 3031

Bruch, A., \& Diaz, M. P. 1998, AJ, 116, 908

Bruch, A., \& Diaz, M. P. 1999, A\&A, 351, 573

Farihi, J., Becklin, E. E., \& Zuckerman, B. 2005, ApJS, 161, 394

Gänsicke, B. T., Araujo-Betancor, S., Hagen, H., et al. 2004, A\&A, 418, 265

Gilmozzi, R., \& Murdin, P. 1983, MNRAS, 202, 587

Haefner, R. 1989, A\&A, 213, L15

Haefner, R., Fiedler, A., Butler, K., \& Barwig, H. 2004, A\&A, 428, 181

Kawka, A., \& Vennes, S. 2003, AJ, 125, 1444

Kawka, A., Vennes, S., Koch, R., \& Williams, A. 2002, AJ, 124, 2853

Kawka, A., Vennes, S., Dupuis, J., Chayer, P., \& Lanz, T. 2008, ApJ, 675, 1518

Kepler, S. O., \& Nelan, E. P. 1993, AJ, 105, 608

Lanning, H. H., \& Pesch, P. 1981, ApJ, 244, 280

Marsh, T. R., \& Duck, S. R. 1996, MNRAS, 278, 565

Maxted, P. F. L., Marsh, T. R., Moran, C., Dhillon, V. S., \& Hilditch, R. W. 1998, MNRAS, 300, 1225

Maxted, P. F. L., O’Donoghue, D., Morales-Rueda, L., Napiwotzki, R., \& Smalley, B. 2007, MNRAS, 376, 919

Morales-Rueda, L., Marsh, T. R., Maxted, P. F. L., et al. 2005, MNRAS, 359, 648

Nelson, B., \& Young, A. 1970, PASP, 82, 699

O’Donoghue, D., Koen, C., Kilkenny, D., et al. 2003, MNRAS, 345, 506

Parsons, S. G., Marsh, T. R., Copperwheat, C. M., et al. 2010, MNRAS, 402, 2591

Parsons, S. G., Marsh, T. R., Gänsicke, B. T., \& Tappert, C. 2011, MNRAS, 412, 2563

Rebassa-Mansergas, A., Gänsicke, B. T., Schreiber, M. R., et al. 2008, MNRAS, 390,1635

Rebassa-Mansergas, A., Gänsicke, B. T., Schreiber, M. R., Koester, D., \& Rodríguez-Gil, P. 2010, MNRAS, 402, 620

Saffer, R. A., Wade, R. A., Liebert, J., et al. 1993, AJ, 105, 1945

Schreiber, M. R., \& Gänsicke, B. T. 2003, A\&A, 406, 305

Shimansky, V., Sakhibullin, N. A., Bikmaev, I., et al. 2006, A\&A, 456, 1069

Sing, D. K., Holberg, J. B., Burleigh, M. R., et al. 2004, AJ, 127, 2936

Somers, M. W., Lockley, J. J., Naylor, T., \& Wood, J. H. 1996, MNRAS, 280, 1277

Taam, R. E., \& Ricker, P. M. 2010, New A Rev., 54, 65

Tappert, C., Gänsicke, B. T., Schmidtobreick, L., et al. 2007, A\&A, 474, 205

Tappert, C., Gänsicke, B. T., Schmidtobreick, L., \& Ribeiro, T. 2011, A\&A, submitted

van den Besselaar, E. J. M., Greimel, R., Morales-Rueda, L., et al. 2007, A\&A, 466, 1031

Webbink, R. F. 2008, in Short-Period Binary Stars: Observations, Analyses, and Results, ed. E. F. Milone, D. A. Leahy, \& D. W. Hobill (Heidelberg: Springer), Astrophys. Space Sci. Libr., 352, 233

Young, A., \& Nelson, B. 1972, ApJ, 173, 653 